\title{
AN UPPER LIMIT TO THE INTRINSIC ANISOTROPY OF THE MICROWAVE BACKGROUND RADIATION ON A SCALE OF 7 ARC MINUTES
}

\author{
A. C. S. Readhead, C. R. Lawrence, S. T. Myers, W. L. W. Sargent \\ Owens Valley Radio Observatory 105-24 \\ California Institute of Technology \\ Pasadena, CA 91125 \\ USA
}

\section{MICROWAVE BACKGROUND RADIATION OBSERVATIONS AT OVRO}

For the last four years we have been engaged on a program to look for intrinsic variations in the Microwave Background Radiation (MBR) at the Owens Valley Radio Observatory (OVRO). We summarize here the results of this continuing search.

The OVRO is well suited to observations of the microwave background radiation since the atmosphere above the site is very dry, and from November until March there is less than $3 \mathrm{~mm}$ of precipitable water above the observatory about $20 \%$ of the time.

The 40 Meter Telescope is used for MBR observations at $20 \mathrm{GHz}$. A superb maser receiver operating at this frequency has been used in the observations reported here. The overall system temperature during good weather is $45-50 \mathrm{~K}$ and the bandwidth is $400 \mathrm{MHz}$. Allowing for the overall beam efficiency of $51 \%$ (including the feeds and telescope), this translates to a sensitivity on the sky of $9 \mathrm{mK} \mathrm{s}^{-1 / 2}$. The receiver is a dual-feed system, with the feeds symmetrically placed on either side of the radio axis. The beam separation on the sky is $7 ! 15$. The receiver has a Dicke switch that operated at $10 \mathrm{~Hz}$ for these observations. In addition to Dicke switching, the beams were alternated on the field of interest. This double switching scheme eliminates linear drifts in sky noise and in ground spillover and linear temperature drifts within the system.

In the search for intrinsic MBR temperature fluctuations the levels of interest are nowadays in the range of tens to hundreds of microKelvin. Thus, even with the best available receivers, many days of observation are needed with the system operating near the thermal noise limit. Over the last four observing seasons 170 days have been allocated to this program at the OVRO.

\section{THE OBSERVATIONS}

Variations in ground spillover are a major source of systematic error in MBR observations with single telescopes. This effect can be minimized by observing fields near the north celestial pole and beam switching only in azimuth. In the present case eight fields situated one degree from the pole were observed. These fields were observed for two-hour periods at upper culmination, thus limiting the change in zenith angle during a scan to 2 arc minutes. Four of the fields were observed at lower culmination in order to look for possible systematic effects. For most of the observations the period of the beam alternation 
was 20 seconds, but for some a period of 40 seconds was used.

Atmospheric temperature variations are another major source of noise. For some of these observations a water vapor radiometer (WVR) was available. This enabled us to measure the atmospheric temperature contribution at $20.7 \mathrm{GHz}$, and to determine the level of random atmospheric fluctuations with sky position. We rejected data for which the scatter was greater than $11 \mathrm{~K}$ per air mass and the scatter in measurements at six zenith angles was greater than $0.4 \mathrm{~K}$. For those periods for which a WVR was not available we kept a careful watch on the weather, and rejected any observations made when clouds (excepting low clouds over the Sierra) were visible within two hours of the observations. Night time observations were rejected if clouds were visible at dawn or dusk.

\section{DATA SELECTION}

There are many sources of noise which can severely corrupt MBR observations. These include instrumental problems, atmospheric effects and ground spillover. Careful data selection is therefore important, since the sensitivity can be improved significantly by eliminating obervations in which one or more of the above effects dominates the noise.

Our data editing procedure consisted of two steps:

1. Hand Editing: Two-hour blocks of data on a single field were rejected if:

i) There was an instrumental failure or the receiver was run on non-standard settings.

ii) The weather was bad, as described above.

iii) The rms scatter in a two-hour block of data was greater than twice the thermal noise limit. Individual points were rejected iteratively if they were more than $3 \sigma$ from the two-hour mean.

$i v)$ The number of data points in a two-hour block of data was less than one third the expected number, and no cause harmless to data quality could be determined.

2. Automatic Editing and Analysis: There is no room here to explain the full details of the automatic analysis, in which individual points are rejected. Our approach is to use both the errors in the individual measurements and the point-to-point scatter to eliminate abnormally noisy points.

We emphasize that our results cannot be biassed in any way by the data selection procedure.

\section{DATA QUALITY}

To reach the sensitivity level needed in intrinsic anisotropy observations any sources of systematic variations above a level of $10 \mu \mathrm{K}$ must be tracked down and eliminated. Our double-switching scheme is a powerful filter against drifts in receiver gain and system temperature, and any signal which survives this filter would either have to be correlated with our 20 second switching cycle or it would have to repeat in a systematic way each time a particular field is observed. Since the fields are observed at upper or lower culmination there is a possibility that diurnal variations in instrumental performance could produce signals with long-term quadratic, or higher order, terms in the switched power which would bias the measurements for particular fields. No systematic diurnal variations were detected, although there are fewer good observations during the day, and therefore these points are noisier.

We have measured very low level variations in the switched power which could introduce a bias at the level of a few microKelvin. This is significantly smaller than the 
level of sensitivity achieved so far, and therefore can have only a very minor effect on our data. Nevertheless, we are taking steps which should reduce this source of error in future observations by at least a factor three.

It is also important to determine the probability distribution of the temperature differences measured. We find that a Gaussian probability distribution fits the data well.

We have also tested the probability that the errors are correlated by by measuring $\sigma^{2}$ as a function of total observing time, $t$. Figure 1 shows the value of $t \sigma^{2} / T_{\text {sys }}^{2}$ as a function of $t$. The point at the extreme right shows the value of $t \sigma^{2} / T_{\mathrm{sys}}^{2}$ for all of the edited data. It is clear from this figure that the errors are uncorrelated up to an integration time of 15 days, and that we are still gaining in sensitivity as $t^{-1 / 2}$.

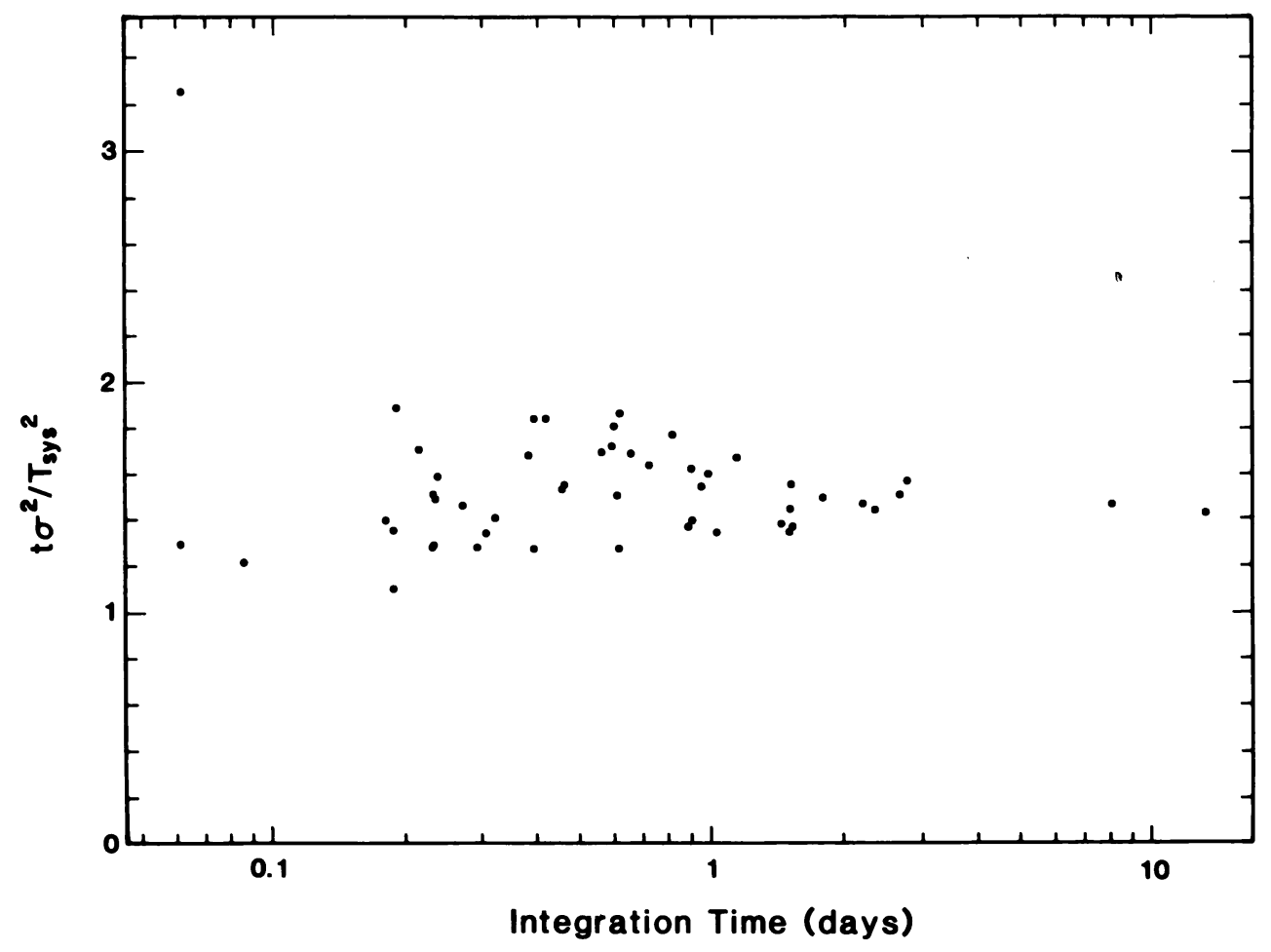

Figure 1. Test for correlated errors. If errors are uncorrelated, we expect $t \sigma^{2} / T_{\text {sys }}^{2}$ to be independent of $t$. It is clear that errors remain uncorrelated up to 16 days.

As a final test of the quality of our data we examined the values of the mean temperature differences for the different epochs. This is the best way to test low level systematic errors that persist for weeks. Data for all fields except Field 7 (see below) have been combined for each session and analyzed by the procedure outlined in the previous section. The reduced $\chi^{2}$ value is 1.7 for 5 degrees of freedom. Assuming a Gaussian distribution, the probability corresponding to a value of $\chi^{2}$ greater than or equal to 1.7 is $12 \%$. This rather low value is due primarily to the low mean for December 1985 . We have made a concerted effort to uncover sources of systematic error which could produce an offset of a few tens of 
microKelvin, but none has been found. The December 1985 point is not so low as to be manifestly due to bad data. Thus our data are consistent with a Gaussian distribution.

The results are shown in Figure 2. Note that one field (Field 7) has a value significantly different from zero. We are fairly confident that this is due to a discrete radio source, and indeed a weak source has been detected near this position (Pauliny-Toth et al. 1977). The measured temperature difference for this field varied significantly between observing epochs, which is further evidence that this is a discrete source. We plan VLA observations to map this field, in order to ascertain that this is indeed a compact extragalactic object. The remaining seven fields are consistent with zero intrinsic fluctuations in the MBR.

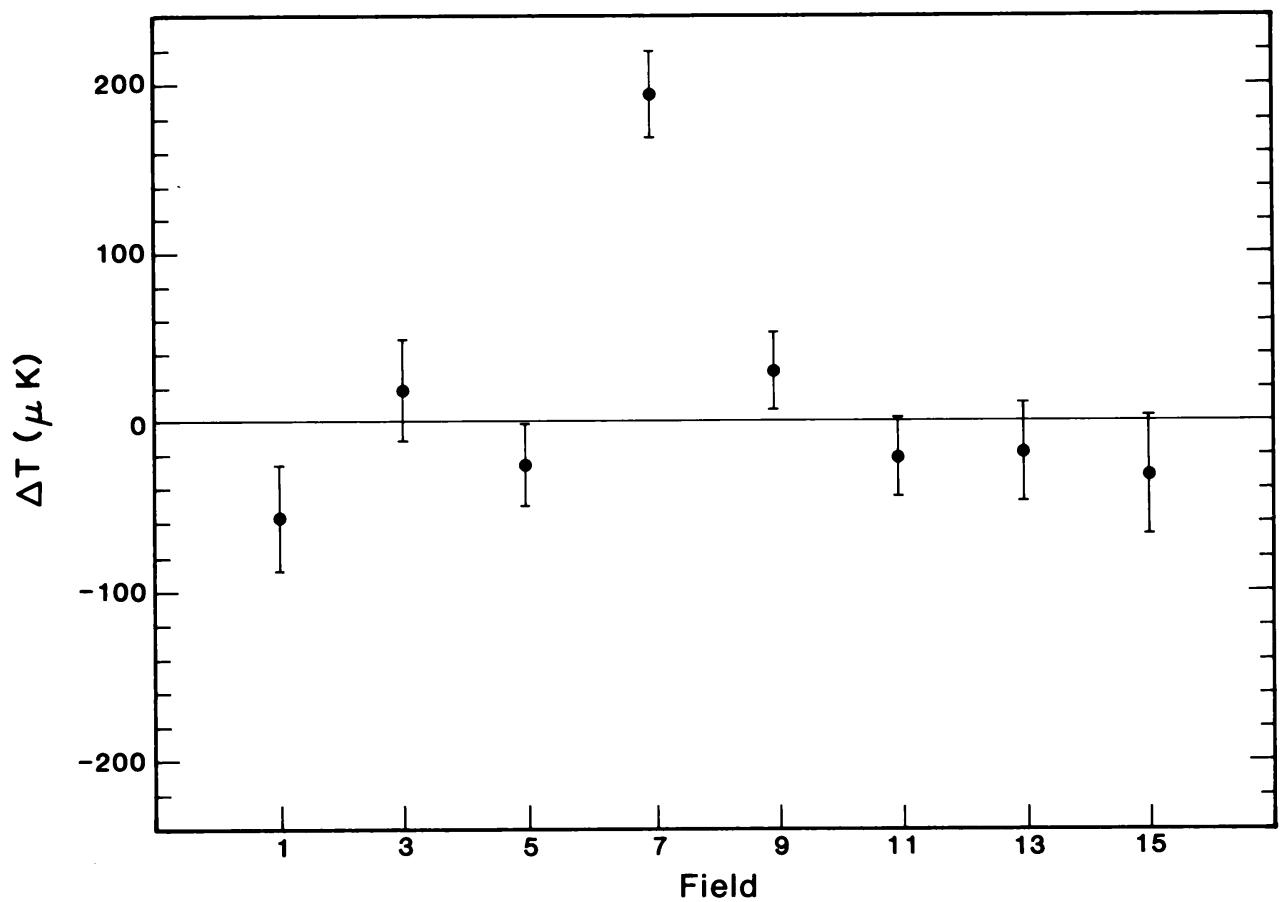

FiguRE 2. Results for eight fields at Decl. $=89^{\circ}$, R.A. $=$ "Field". Error bars are based on the scatter of the individual $80 \mathrm{~s}$ data points.

\section{DATA ANALYSIS}

A likelihood ratio test provides a uniformly most powerful test for an upper limit to the intrinsic anisotropy based on our measurements. Applying this, by calculating the distribution of the required sufficient statistic using Monte Carlo methods, yields an upper limit of $47 \mu \mathrm{K}(95 \%)$, which translates to an upper limit for on the 7.15 scale of $\Delta T / T=1.4 \times 10^{-5}$. (Note that there is a factor of $\sqrt{2 / 3}$ due to our double switching 
scheme.) Alternatively, a Bayesian analysis with a uniform prior density yields $51 \mu \mathrm{K}$ at the $95 \%$ level, corresponding to an upper limit of $\Delta T / T=1.5 \times 10^{-5}$.

\section{DISCUSSION}

These limits rule out adiabatic fluctuations and most isothermal fluctuation models. They are consistent with cold dark matter models of the types considered by Bond and Efstathiou (1987). Since our sensitivity is still increasing as $t^{-1 / 2}$, and the current limit is based on 15 days of data, two additional winters with good weather could yield a factor of two improvement in our results. At this level, discrete-source confusion may prove to be the biggest difficulty in interpretation.

Early in 1988 a new instrument, dedicated to microwave background observations, will begin operating at OVRO. A $5.5 \mathrm{~m}$ antenna will have a $32 \mathrm{GHz}$ maser receiver with expected sensitivity on the sky of about $5 \mathrm{mK} \mathrm{s}^{-1 / 2}$. The beamwidth will be about $8^{\prime}$, and the beam separation about $24^{\prime}$. A $1 \mathrm{kHz}$ Dicke switch, built-in cold loads, symmetrical internal configuration, and a digital back end should provide us with better control over systematic errors than the $40 \mathrm{~m}$ system. With its increased sensitivity and larger beam separation, this new instrument will be the key instrument in the MBR program at OVRO.

Acknowledgements: We would like to thank Harry Hardebeck and Al Moffet for their essential contributions to this work. Microwave background research at OVRO is supported by the IBM Research Fund, the Irvine Foundation, and NSF grants AST 85-09822 and AST 86-10693.

\section{REFERENCES}

Bond, J. R., and Efstathiou, G. 1987, M. N. R. A. S., 226, 655.

Pauliny-Toth, I. I. K., Witzel, A., Preuss, E., Baldwin, J. E., and Hills, R. E. 1977, Astron. Astrophys. Suppl., 34, 253. 